\title{
Continuous Wavelet Transform Based Gene Optimized Fuzzy C-Means Clustering For Forest Fire Detection
}

\author{
Pushpa Balasubramanian, Kamarasan Mari
}

\begin{abstract}
Fire detection is an important aspect of disaster preparedness, to reduce loss of lives and property damage. Conventionally, many techniques have been designed so far, to discover the forest fires through input videos. But, clustering performance of conventional fire detection techniques was not sufficient. To overcome the above limitations, Continuous Wavelet Transform Based Gene Optimized Fuzzy C-Means Clustering (CWT-GOFCC) technique is proposed. The proposed CWT-GOFCC technique takes number of video files from FIRESENSE database as input and converts those input videos into a number of frames. Next, it defines the number of clusters and centroids and consequently initializes the gene populations with number of video frames. After that, CWT-GOFCC technique evaluates fuzzy membership with the assistance of fitness function for all input video frames based on spatial correlation between the fire flame colors. By using this fitness function, the technique groups the video frames into pre-fire stage or fire stage or critical fire stage with enhanced accuracy. From that, this technique accurately clusters all the video frames into related clusters with lower time consumption. The simulation of the technique is conducted using metrics such as fire detection accuracy, fire detection time and false positive rate with respect to different numbers of video frames. The simulation result depicts that the technique is able to improve the accuracy and also reduce the time of forest fire detection in video file when compared to state-of-the-art works.
\end{abstract}

Index Terms: Continuous Wavelet Transform, Fire detection, Fire Flame Colors, Fitness function, Fuzzy Membership, Spatial Correlation, Video Frames.

\section{INTRODUCTION}

Fire detection in videos is essential for early discovery of fire. Video-based Fire detection systems identify uncontrolled fires at an early stage before they turn into catastrophic incidents. A lot of research works have been developed for performing fire detection in video. But, clustering performance of fire detection was lower which increases a false positive rate. Hence, CWT- GOFCC technique is designed in this research work with application of Continuous Wavelet transformation, Genetic optimization

Revised Manuscript Received on July 08, 2019.

PUSHPA BALASUBRAMANIAN, Computer and Information Science, Annamalai University, Annamalainagar, 608002 India. amst1920@ gmail.com

KAMARASAN MARI, Computer and Information Science, Annamalai University, Annamalainagar, 608002 India. and Fuzzy C-

Means clustering to increase the accuracy of detecting forest fires in input video.

An ICA Kmedoids-based color model was designed in [1] for obtaining high detection accuracy and a low false detection rate. But, the amount of required for detecting the forest fire in a given video was more. Spatial fuzzy C-means clustering (SpFCM) was presented in [2] for fire detection using visible and infrared fire images with a lower time. However, clustering performance of SpFCM was lower which impact the fire detection accuracy. Light Condition determination was employed in [3] to perform video fire discovery in Spacious Buildings. But, time complexity was higher. Spatio-Temporal Flame Modeling and Dynamic Texture Analysis was employed in [4] for fire detection system with high true positives. However, fire detection accuracy was lower.

An improved probabilistic approach was presented in [5] with application of two improved feature representations for color-based fire detection. But, false positive rate of fire detection was not solved. A Spatio Wildfire Prediction and Monitoring System (SWPMS) were introduced in [6] to detect flames in forest with higher accuracy. However, computational complexity involved during fire detection process was not reduced. Dirichlet Process Gaussian Mixture Model based approach was used in [7] for autonomous flame discovery. But, ratio number of video frames inaccurately detected was more. Principal component analysis (PCA) was designed in [8] to increases performances of real-time forest fire detection. However, fire detection performance was poor.

In order to resolve the above said existing drawbacks, CWT-GOFCC technique is introduced. The key contributions of this technique are explained below.

- To increase the performance of forest fire detection as compared to state-of-the-art works, CWT-GOFCC technique is proposed. Contrary to the conventional works, this technique is intended with aid of Continuous Wavelet Transformation, Genetic algorithm in Fuzzy C-Means Clustering.

- To improve the accuracy of forest fire detection, color auto correlgram is employed in this technique. The auto color correlogram measures spatial

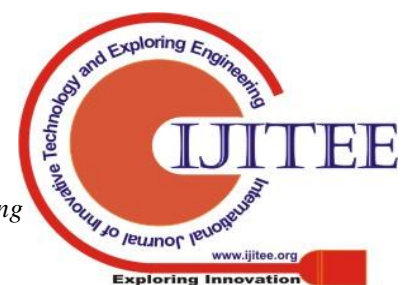


correlation between colors by considering both the global and local distributions of colors in input video frames. The auto color correlogram in CWT-GOFCC technique is robust in tolerating large changes in the appearance of a scene caused by changes in viewing positions, changes in the background scene. This supports for CWT-GOFCC technique to efficiently find the forest fires in video.

The residual paper structure is created as follows. Section II shows the related works. Section III explains the detailed process of CWT-GOFCC technique. In section IV, a simulation setting of the proposed technique is described. Section $\mathrm{V}$ presents the results and discussion of this technique using parameters. Finally, the conclusion of the paper is discussed in Section VI.

\section{RELATED WORKS}

Efficient color based fire pixel segmentation was employed in [9] for separating fire flame pixels and thereby enhancing forest fire detection accuracy. $\mathrm{L}^{*} \mathrm{a} * \mathrm{~b} * \mathrm{YCbCr}$ color space and k-means clustering algorithm was presented in [10] for forest fire recognition. K-medoids Clustering was designed in [11] to obtain better fire flame prediction accuracy. A novel forest fire detection system was utilized in [12] with application of background subtraction and color segmentation. Real-time multi-characteristic based fire flame recognition was designed in [13] to get higher the reliability. Spatio-Temporal Flame Modeling and Dynamic Texture examination was applied in [14] to discover fire flame in video frames.

\section{CONTINUOUS WAVELET TRANSFORM BASED GENE OPTIMIZED FUZZY C-MEANS CLUSTERING TECHNIQUE}

The Continuous Wavelet Transform Based Gene Optimized Fuzzy C-Means Clustering (CWT-GOFCC) technique is proposed to perform efficient forest fire flame detection with a minimal time complexity through clustering. The procedure is introduced by application of Continuous Wavelet Transformation, Genetic algorithm in Fuzzy C-Means Clustering on the contrary to conventional works. The Continuous Wavelet Transform is applied in this technique to decompose a video frames into a number of sub-bands. On the contrary to existing works, Continuous Wavelet Transform makes a time-frequency representation of a video frames that offers very good image quality for finding the forest fires.

In addition to that, genetic optimization algorithm is employed in Fuzzy C-Means Clustering to measure the fuzzy membership for each input video frames with the help of fitness function using color auto correlgram. In this technique, color auto correlgram captures only the color distribution in an input video frames. On the contrary to local properties such as pixel position, gradient direction, or global properties, color auto correlgram identifies the spatial correlation among fire flame colors. This helps for CWT-GOFCC technique to cluster the video frames into a different clusters i.e. pre-fire stage, fire stage and critical fire stage. The architecture diagram of CWT-GOFCC technique is depicted in below Fig.1.

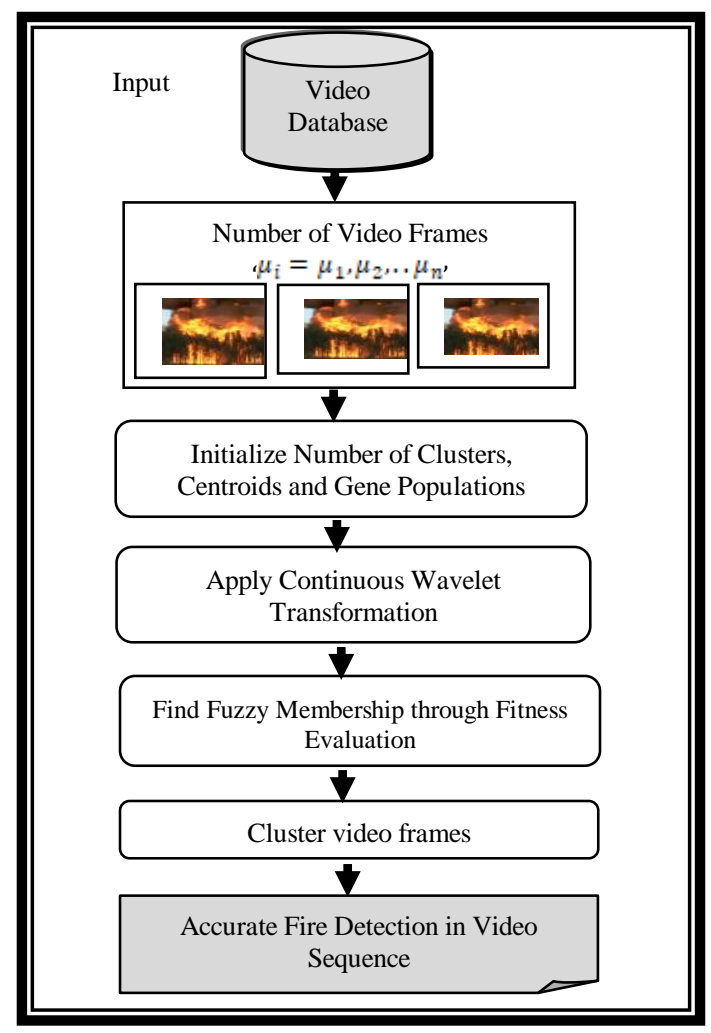

\section{Fig. 1. Architecture Diagram of CWT-GOFCC Technique for Forest Fire Detection}

Fig.1 demonstrates the overall processes of CWT-GOFCC technique to increase performance of forest fire flame in video sequence. As shown in above fig, this technique at first gets video database (i.e. FIRESENSE database) as input. The input video file comprises of many video frames. Therefore, the proposed technique converts video file to number of frames. After that, this technique defines initializes of clusters, centroids and gene populations by considering number of input video frames. Next this approach used Continuous Wavelet Transformation to divide the each video frames into number of sub-bands. Subsequently, the technique measures fuzzy membership using fitness evaluation based on spatial correlation between fire flame colors. Finally, cluster the video frames into a pre-fire stage, fire stage and critical fire stage according to estimated fitness function. From that, CWT-GOFCC technique attains improved accuracy for 
forest fire flame detection as compared to existing works. The detailed process of CWT-GOFCC technique is explained in below.

Let us assume an input video database contains numerous videos file denoted as ' $\rho_{i}=\rho_{1}, \rho_{2}, \ldots, \rho_{n}$ '. Here, ${ }^{~} n$, indicates total number of video files presented in input database. The input video file shown in below Fig. 2.

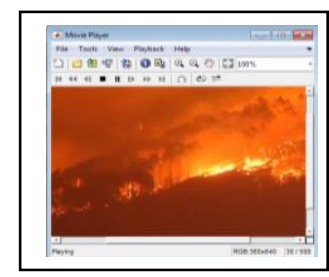

Fig. 2. Input Video File

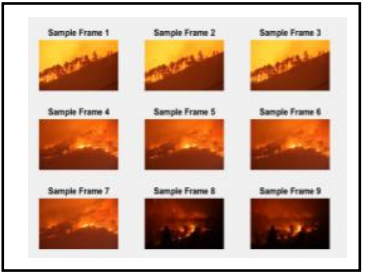

Fig. 3. Input Video File
The video files are transformed into number of frames represetned as ' $\mu_{i}=\mu_{1}, \mu_{2}, \ldots \mu_{N}$ ' whereas ${ }^{\prime} N$, point outs the number of frames as depicted in above fig.3. The proposed technique at first initializes number of clusters and cluster centroids. Subsequently, this technique initialize gene populations ' IGP, with help of number of video frames using below mathematical expression,

$$
I G P=\mu_{1}, \mu_{2}, \ldots \mu_{N}
$$

From the above equation (1), ${ }^{N}$, indicates a number of video frames in input video file. The CWT-GOFCC technique process begins with a set of individuals (i.e video frames) which is called a Population. Each individual is a solution to the problem. In this technique, the video frame is represented by a set of parameters called as Genes. Genes are combined into a string to make a chromosome.

Then, continuous wavelet transform is applied in CWT-GOFCC technique to split the images into a number of sub-blocks. The Continuous wavelet transform (CWT) is utilized to partition a continuous-time function into wavelets. Therefore, continuous wavelet transform of a video frame ' $\mu_{i}$, at a scale ' $(a>0)$, and translational value $b \in R$, is mathematically perofrmed as,

$$
\mu_{w(a, b)}=\frac{1}{|a|^{\frac{1}{2}}} \int_{-\infty}^{\infty} \mu(t) \bar{\psi}\left(\frac{t-b}{a}\right) d t
$$

From the above equation (2), $\psi(\psi(t)$, indicates the continues function in both the time domain and and the frequency domain and the overline denotes complex conjugate operation. The continuous wavelet transform of a video frames are presented in below Fig. 4 .
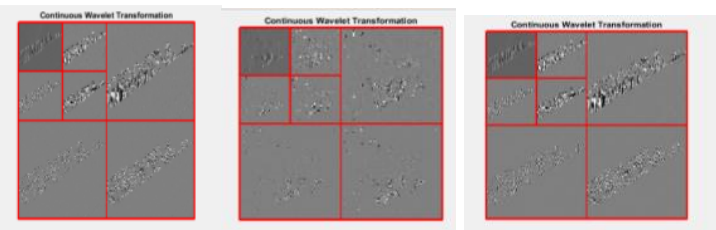

After transformation process, the technique computes fuzzy membership via fitness function evaluation for each input video frame ' $\mu_{i}$, . On the contrary to state-of-the-art works, fuzzy membership is determined in this technique using fitness function which assists to exactly cluster input video frames with higher accuracy and minimal time.

In CWT-GOFCC technique, the fuzzy membership is estimated through fitness function based on spatial correlation between fire flame colors in a given video frame ، $\mu_{i}$, using below,

$$
\text { Fuzzy } y_{m e m}=\alpha_{\mu_{i}}
$$

From the above equation (3), 'Fuzzy ${ }_{m e m}$ ' denotes the fuzzy membership whereas ${ }^{~} \alpha_{\mu_{i}}$, represents the fitness function determined for each video frame ' $\mu_{i}$ '. The Fuzzy membership calculation for different video frames is shown in below.
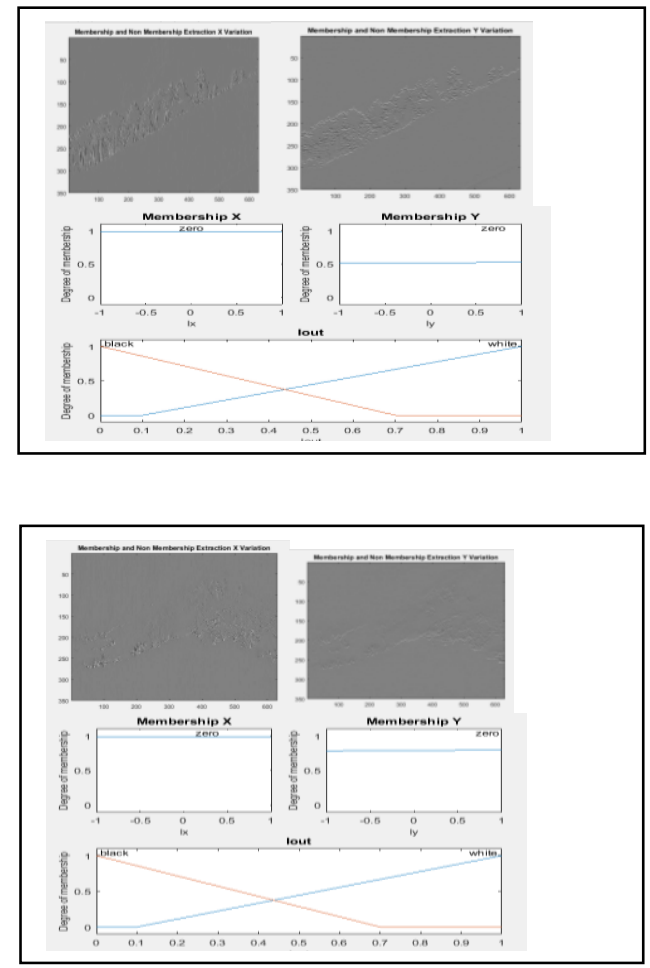

Fig. 4 continuous wavelets transform

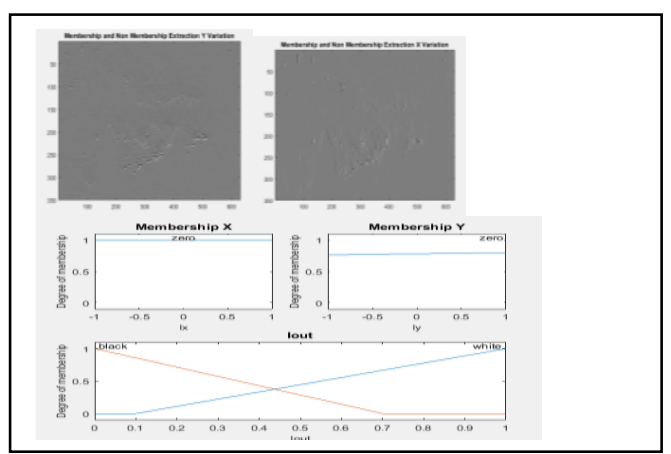

Fig. 5. Fuzzy membership 


\section{calculation of different} video frames

In this technique, Fuzzy membership is estimated with assists of fitness function using color auto correlgram. On the contrary to conventional works, the proposed technique used color auto correlgram for effective forest fire dectection. An autocorrelogram finds spatial correlation between fire flame colors. Because, correlations between colors are more important to accurately detect the forest fires. This supports for efficently group the video frames into a corresponding clusters.

The correlogram of the video frame ' $\mu_{i}$ ' is measured for color pair ${ }^{~} c_{i,} C_{j}$, at a distance ${ }^{~} \tau$, using below mathematical representation,

$$
z_{c_{i,} c_{j}}^{\tau}\left(\mu_{i}\right)=\operatorname{Pr} r_{i \in \mu_{c_{i}} j} j \in \mu\left[j \in \mu_{c_{j}}|i-j=\tau|\right]
$$

From the above equation (4) given any pixel of color ' $c_{i_{x}}$ ' in video frame, ${ }^{2} z_{c_{i_{2}}}^{\tau} c_{j}\left(\mu_{i}\right)$, represents the probability that a pixel at distance ' $\tau$, away from the given pixel is of color ' $c_{j}$,

Autocorrelogram determines the spatial correlation of identical colors using below mathematical representation,

$$
\beta_{c}^{\tau}\left(\mu_{i}\right)=z_{c_{i}, c_{j}}^{\tau}\left(\mu_{i}\right)
$$

From the above equation (5), ' $\beta_{c}^{\tau}\left(\mu_{i}\right)$, finds the spatial correlation between fire flame colors in each input video frame ' $\mu_{i}$, Based on the measured spatial correlation between identical colors, then CWT-GOFCC technique determines fitness function for each input video frame ${ } \alpha_{\mu_{i}}$, using below mathematical expression,

$$
\alpha_{\mu_{i}}=\beta_{c}^{\tau}\left(\mu_{i}\right)
$$

From equation (6), fitness function is calculated for all video frames ' $\mu_{i}$, in a given video file. Subsequently, this technique set threshold value for fitness function. In CWT-GOFCC technique, threshold value is predefined for spatial correlation between fire flame colors to increases the clustering accuracy of forest fire detection. Thus, the technique groups the input video frames into a corresponding clusters using below mathematical formula,

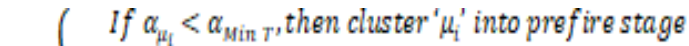

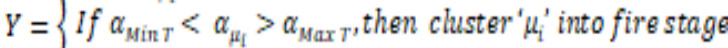 \\ If $\alpha_{\mu_{\mathrm{P}}}<\alpha_{\mathrm{Max}}$, then cluster " $\mu_{\mathrm{P}}$ into critical fire stage}

From the equation (7), ' $Y$, denotes a clustered result of video frame ' $\mu_{i}$,. Here, “ ${ }^{\alpha} \mu_{i}$ ' represents a measured fitness function value of video frame whereas ${ }^{\prime} \mu_{i}$ ' point outs an ${ }^{i}{ }^{t h}$, frame in given video sequence and ' $\alpha_{\operatorname{Min} T}$, indicates a minimum threshold fitness function and ' $\alpha_{\operatorname{MaxT}}$ ' is a maximum threshold fitness function. By using the above equation, CWT-GOFCC technique enhances the clustering performance to effectively identify the forest fires in video with a minimal time as shown in below fig.

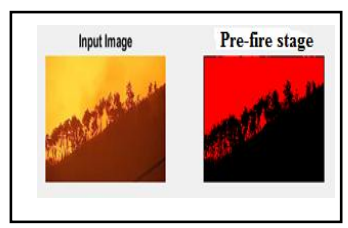

b) Pre-fire stage

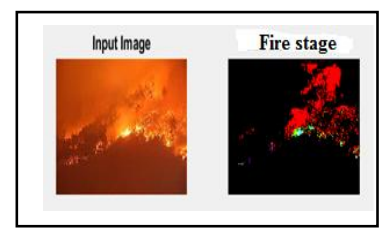

a) fire stage

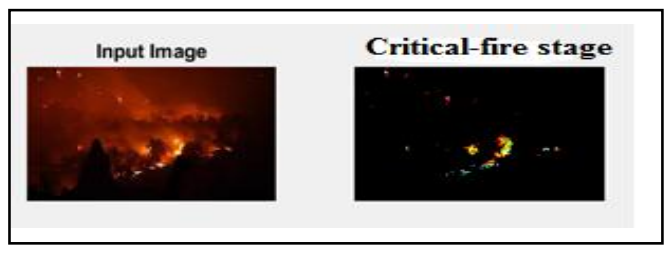

c) Critical-fire stage

\section{Fig. 6. Clustering of video frames}

The above fig.6 demonstrates the examples for clustering of input video frames into a pre-fire, fire and critical fire stages. The algorithmic step of proposed CWT-GOFCC technique is explained in below. In algorithm 1 shows step by step process of this technique to achieve enhanced clustering performance for forest fire detection. By using the below algorithmic process, CWT-GOFCC technique determines fuzzy membership for each video frames using fitness function according to spatial correlation between fire flame colors. This supports for the proposed technique to significantly cluster the video frames into a diverse clusters i.e. pre-fire, fire and critical stage with higher accuracy and a lower time. Hence, the technique provides better performance in terms of accuracy, time and false positive rate for forest fire detection as compared to conventional works. 


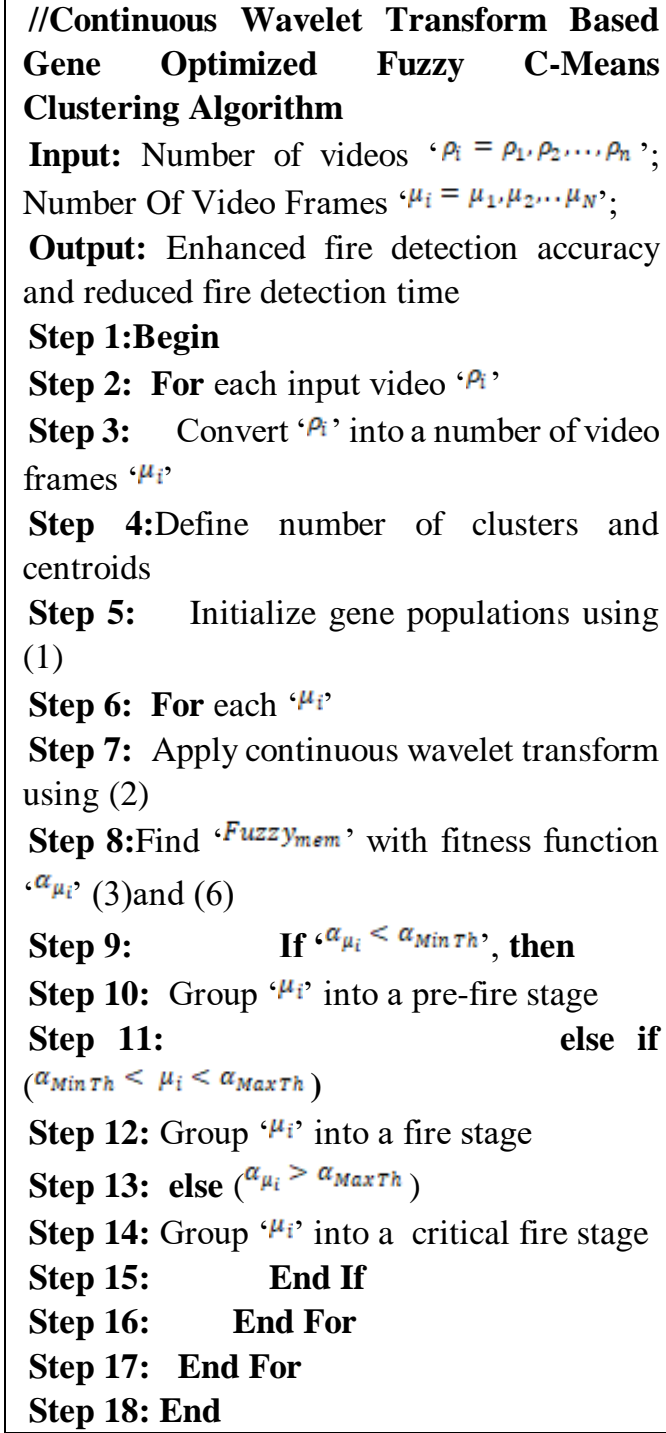

Algorithm 1. Continuous Wavelet Transform Based GeneOptimized Fuzzy C-Means Clustering

\section{SIMULATION SETTINGS}

The CWT-GOFCC technique is implemented in MATLAB using FIRESENSE database [15] to determine proposed performance. To perform the simulation work, the proposed technique takes different numbers of video frames in the range of 25-250 from FIRESENSE database. The efficiency of this technique is determined in terms of fire detection accuracy, fire detection time and false positive rate. The performance result of this technique is compared with two conventional works namely ICA (Imperialist Competitive Algorithm) Kmedoids-based color model [1] and spatial fuzzy C-means clustering (SpFCM) [2].

\section{RESULT AND DISCUSSIONS}

In this section, the comparative result analysis of CWT-GOFCC technique is discussed using parameters such as fire detection accuracy, fire detection time and false positive rate with the assist of below graphical representations.

\section{A. Simulation Measure Of Fire Detection Accuracy}

Fire Detection Accuracy ' $F D A$, estimate the ratio of number of video frames accurately clustered to the total number of video frames as input. The fire detection accuracy is determined using below,

$$
F D A=\frac{n_{A C}}{N} * 100
$$

From equation (8), ' $n_{A C}$ ' signifies number of video frames correctly clustered whereas ' $N$, denotes a total number of video frames taken for simulation process. The fire detection accuracy is measured in terms of percentages (\%). The comparative result analysis of the detection accuracy is presented in below Table I.

Table I. Tabulation Result of Fire Detection Accuracy

\begin{tabular}{|l|l|l|l|}
\hline \multirow{2}{*}{$\begin{array}{l}\text { Number of } \\
\text { Video Frames }\end{array}$} & \multicolumn{3}{|c|}{ Fire Detection Accuracy (\%) } \\
\cline { 2 - 4 } & CWT-GOFCC & $\begin{array}{l}\text { ICA Kmedoids-based } \\
\text { color model }\end{array}$ & SpFCM \\
\hline 25 & 92 & 80 & 68 \\
\hline 50 & 94 & 82 & 78 \\
\hline 75 & 95 & 83 & 77 \\
\hline 100 & 93 & 85 & 79 \\
\hline 125 & 96 & 84 & 81 \\
\hline 150 & 94 & 87 & 77 \\
\hline 175 & 96 & 87 & 83 \\
\hline 200 & 92 & 85 & 81 \\
\hline 225 & 94 & 86 & 86 \\
\hline 250 & 97 & 88 & \\
\hline
\end{tabular}

Fig. 7 shows impact of fire detection accuracy versus different numbers of video frames using three methods namely CWT-GOFCC technique, ICA Kmedoids-based color model [1] and SpFCM [2]. As demonstrated in above fig, the proposed CWT-GOFCC technique obtains higher fire detection accuracy when compared to existing ICA Kmedoids-based color model [1] and SpFCM [2]. This is because of continues wavelet transformation, genetic algorithm and fuzzy c-means clustering process combined in CWT-GOFCC technique on the contrary to existing algorithms.

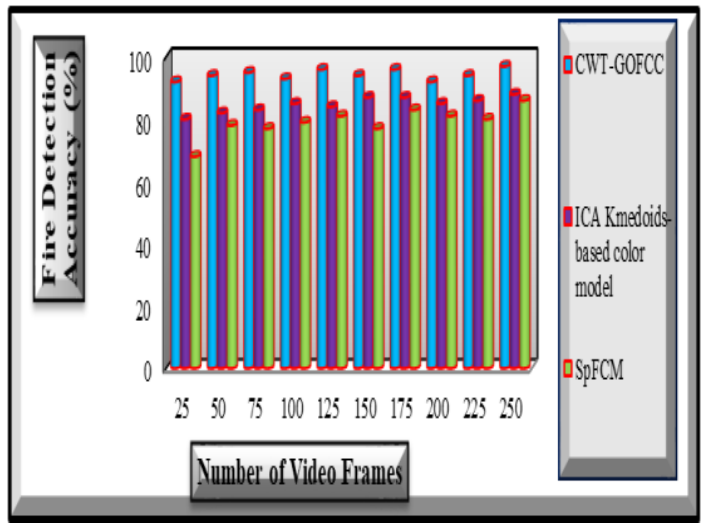

Fig.7. Measurement of Fire Detection Accuracy versus Number of Video Frames

In CWT-GOFCC technique, 
video frames' clustering is performed based on estimated spatial correlation between fire flame colors. This supports for the technique to enhance the ratio of number of video frames that are exactly clustered when compared to other conventional works. Thus, the technique improves the fire detection accuracy by $11 \%$ as compared to K ICA Kmedoids-based color model [1] and $20 \%$ as compared to SpFCM [2].

\section{B. Simulation Measure of Fire Detection Time}

Fire Detection Time 'FDT, determines amount of time utilized to cluster input video frames. The fire detection time is mathematically evaluated using below,

$$
F D T=N \text { time }(C S)
$$

From equation (9), 'time (CS), point outs the time employed for clustering fire flame in single video frame and ' $N$, indicates number of video frames considered for simulation work. The fire detection time is calculated in terms of milliseconds (ms). The performance result analysis of fire detection time is demonstrated in below Table II.

\section{Table II. Tabulation Result of Fire Detection Time}

\begin{tabular}{|c|c|c|c|}
\hline \multirow{2}{*}{\begin{tabular}{l}
\multicolumn{2}{c}{ Num } \\
ber of \\
Video \\
Frames
\end{tabular}} & \multicolumn{3}{|c|}{ Fire Detection Time (ms) } \\
\hline & ${ }_{C C} C W T-G O F$ & $\begin{array}{c}\text { ICA } \\
\text { Kmedoids-base } \\
\text { d color model }\end{array}$ & SpFCM \\
\hline 25 & 26 & 33 & 34 \\
\hline 50 & 28 & 34 & 36 \\
\hline 75 & 32 & 42 & 46 \\
\hline 100 & 35 & 46 & 51 \\
\hline 125 & 41 & 56 & 63 \\
\hline 150 & 44 & 62 & 69 \\
\hline 175 & 47 & 68 & 77 \\
\hline 200 & 50 & 70 & 82 \\
\hline 225 & 54 & 77 & 83 \\
\hline 250 & 58 & 83 & 85 \\
\hline
\end{tabular}

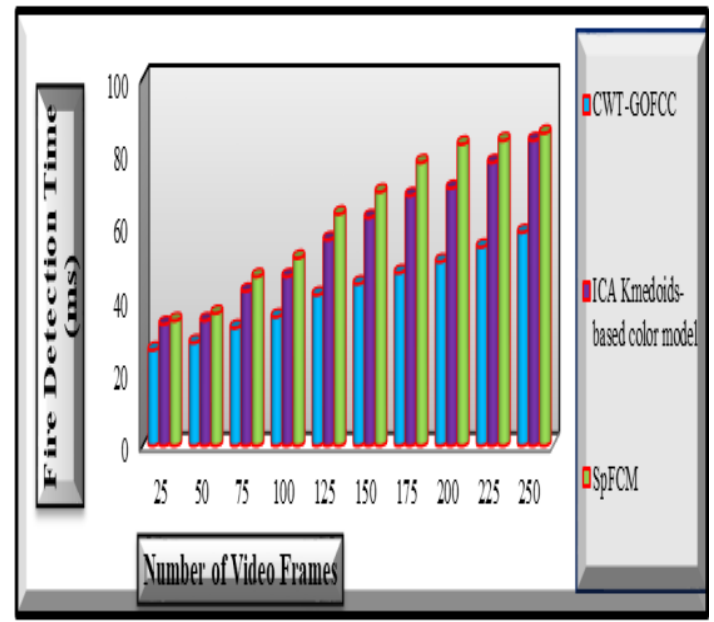

Fig. 8. Measurement of Fire Detection Time versus Number of Video Frames

Fig. 8 illustrates impact of fire detection time versus varied numbers of video frames using three methods namely CWT-GOFCC technique, ICA Kmedoids-based color model [1] and SpFCM [2]. As presented in above fig, the proposed CWT-GOFCC technique gets lower fire detection time as compared to existing ICA Kmedoids-based color model [1] and SpFCM [2]. This is owing to application of continues wavelet transformation, genetic optimization algorithm and fuzzy c-means clustering in this technique on the contrary to conventional methods.

CWT-GOFCC technique utilizes fitness function for finding fuzzy membership for each input video frames. This helps for the technique to utilize a minimal amount of time to precisely find the different types of forest fires through clustering as compared to other existing works. Therefore, CWT-GOFCC technique reduces the fire detection time by $26 \%$ as compared to ICA Kmedoids-based color model [1] and $32 \%$ while compared to SpFCM [2].

\section{Simulation Measure of False positive Rate}

False Positive Rate 'FPR' measures the ratio of number of video frames inaccurately clustered to the total number of video frames as input. The false positive rate is mathematically obtained as,

$$
F P R=\frac{n_{W C}}{N} * 100
$$

From equation (10), the false positive rate of fire flame detection is measured. Here, ' $n_{W C}$ ' represents number of frames that are inaccurately clustered and $~ N$, denotes a total number of video frames. The false positive rate is evaluated in terms of percentage (\%).The simulation result analysis of false positive rate is presented in below Table III.

\begin{tabular}{|c|c|c|c|}
\hline \multirow{2}{*}{$\begin{array}{l}\text { Num } \\
\text { ber of } \\
\text { Video } \\
\text { Frames }\end{array}$} & \multicolumn{3}{|c|}{ False positive Rate (\%) } \\
\hline & $\begin{array}{l}C W T-G O F \\
C C\end{array}$ & $\begin{array}{c}\text { ICA } \\
\text { Kmedoids-base } \\
\text { d color model }\end{array}$ & $M^{S p F C}$ \\
\hline 25 & 8 & 20 & 32 \\
\hline 50 & 6 & 18 & 22 \\
\hline 75 & 5 & 17 & 23 \\
\hline 100 & 7 & 15 & 21 \\
\hline 125 & 4 & 16 & 19 \\
\hline 150 & 6 & 13 & 23 \\
\hline 175 & 4 & 13 & 17 \\
\hline 200 & 8 & 16 & 19 \\
\hline 225 & 6 & 14 & 20 \\
\hline 250 & 3 & 12 & 14 \\
\hline
\end{tabular}

Table III. Tabulation Result of False positive Rate

Fig. 9 depicts the impact of false positive rate versus diverse numbers of video frames using three methods namely CWT-GOFCC technique,

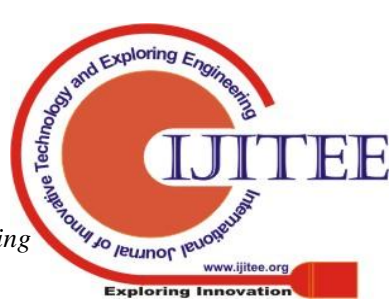


ICA Kmedoids-based color model [1] and SpFCM [2]. As exposed in below fig, the proposed CWT-GOFCC technique obtains a minimal false positive rate for discovering the forest fires in video when compared to existing ICA Kmedoids-based color model [1] and SpFCM [2].

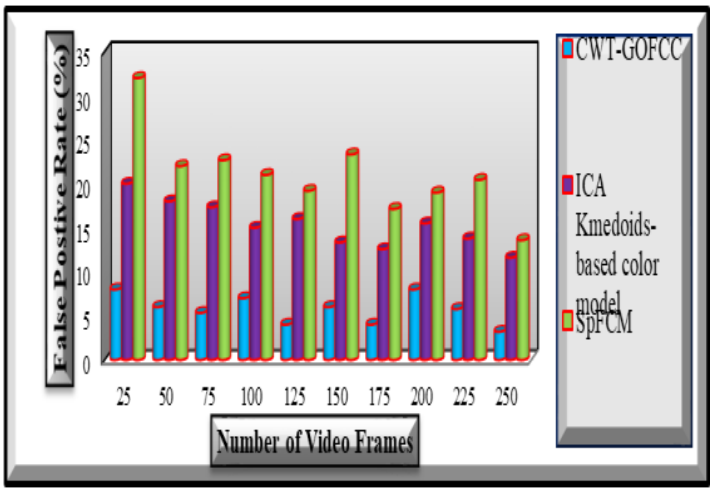

Fig. 9. Measurement of False positive Rate versus number of Video Frames

This is due to application of continues wavelet transformation, genetic optimization algorithm and fuzzy c-means clustering in CWT-GOFCC technique on the contrary to state-of-the-art algorithms where it computes fuzzy membership using fitness function by considering spatial correlation between fire flame colors. From that, this technique accomplishes effective clustering. Hence, the ratio of number of frames that are wrongly clustered using CWT-GOFCC technique is minimized when compared to other existing works. As a result, CWT-GOFCC technique decreases the false positive rate by $63 \%$ as compared to ICA Kmedoids-based color model [1] and $73 \%$ while compared to SpFCM [2].

\section{CONCLUSION}

The CWT-GOFCC technique is developed with the objective of increasing the performance of forest fire detection in video sequence. This objective is achieved by integrating continuous wavelet transformation, genetic optimization algorithm and fuzzy c-means clustering. The designed CWT-GOFCC technique minimizes the time consumed to correctly cluster the video frames as pre-fire, fire and critical fire stages when compared to the other contemporary works. In addition, the technique enhances the clustering accuracy of fuzzy c-means using genetic algorithm. Thus it helps to decrease the number of video frames that are wrongly clustered. The performance of CWT-GOFCC technique is estimated in terms of fire detection accuracy, fire detection time, and false positive rate and compared with two existing works. The experimental result illustrates that CWT-GOFCC technique presents better performance with an improvement of fire detection accuracy and minimization of fire detection time when compared to conventional works.

\section{REFERENCES}

1. Mahdi Hashemzadeh, Alireza Zademehdi, "Fire detection for video surveillance applications using ICA K-medoids-based color model and efficient spatio-temporal visual features", Expert Systems with Applications, Elsevier, Volume 130, Pages 60-78, September 2019.

2. Siva Mouni Nemalidinne, Deep Gupta, "Nonsubsampled contourlet domain visible and infrared image fusion framework for fire detection using pulse coupled neural network and spatial fuzzy clustering", Fire Safety Journal, Elsevier, Volume 101, Pages 84-101, October 2018.

3. Yang Jia, Gaohua Lin, Jinjun Wang, Jun Fang, Yongming Zhang, "Light Condition Estimation Based on Video Fire Detection in Spacious Buildings", Arabian Journal for Science and Engineering, Springer, Volume 41, Issue 3, Pages 1031-1041, March 2016.

4. P.Tamil Mathi, Dr. L. Latha, "Video Based Forest Fire Detection using Spatio-Temporal Flame Modeling and Dynamic Texture Analysis", International Journal on Applications in Informaton and Communication Engineering Volume 2, Issue 4, Pages 41-47, April 2016

5. Zhijie Zhang, Tian Shen, Jianhua Zou, "An Improved Probabilistic Approach for Fire Detection in Videos", Fire Technology, Springer, Volume 50, Issue 3, Pages 745-752, May 2014.

6. Gudikandhula Narasimha Rao, Peddada Jagadeeswara Rao, Rajesh Duvvuru, Sridhar Bendalam, Roba Gemechu, "An enhanced real-time forest fire assessment algorithm based on video by using texture analysis", Perspectives in Science, Elsevier, Volume 8, Pages 618-620, September 2016.

7. Zhenglin Li, Lyudmila S Mihaylova, Olga Isupovaand Lucile Rossi, "Autonomous Flame Detection in Videos with a Dirichlet Process Gaussian Mixture Color Model", Journal Of Industrial Informatics, Special Section Of Multisensor Fusion And Integration For Intelligent Systems, Volume 14, Issue 3, Pages 1146-1154, 2017.

8. Shixiao $\mathrm{Wu}$ and Chengcheng Guo, "Using Combination Methods to Improve Real Time Forest Fire Detectio n", Journal of Statistics and Mathematical Sciences, Volume 5, Issue 1, Pages 1-10, December 2018

9. C. E. Prema et al, "Efficient Colour Based Fire Pixel Classification Using Image Processing", Applied Mechanics and Materials, Volume 626, Pages 52-57, 2014

10. Rui Chen, Yuanyuan Luo, Mohanmad Reza Alsharif, "Forest Fire Detection Algorithm Based On Digital Image", Journal of Software, Volume 8, Issue 8, Pages 1897- 1905, August 2013.

11. Amin Khatami, Saeed Mirghasemi, Abbas Khosravi, Chee Peng Lim, Saeid Nahavandi, "A new PSO-based approach to fire flame detection using K-Medoids clustering”, Expert Systems with Applications, Volume 68, Pages 69-80, February 2017.

12. Mubarak A. I. Mahmoud, and Honge Ren, "Forest Fire Detection Using a Rule-Based Image Processing Algorithm and Temporal Variation”, Mathematical Problems in Engineering, Volume 2018, Article ID 7612487, Pages 1-8, 2018,

13. Rui Chi, Zhe-Ming Lu, Qing-Ge Ji, "Real-time multi-feature based fire flame detection in video",ET Image Processing, Volume 11, Issue 1 , Pages $31-37,2017$.

14. Kosmas Dimitropoulos, Panagiotis Barmpoutis and Nikos Grammalidis, "Spatio-Temporal Flame Modeling and Dynamic Texture Analysis for Automatic Video-Based Fire Detection", IEEE Transactions on Circuits and Systems for Video Technology (TCSVT), Volume 25, Issue 2, Pages 339-351, February 2015.

15. FIRESENSEdatabase: https://zenodo.org/record/836749

\section{AUTHORS PROFILE}

PUSHPA BALASUBRAMANIAN, Computer and Information Science, Annamalai University, Annamalainagar, 608002 India.

KAMARASAN MARI, Computer and Information Science, Annamalai University, Annamalainagar, 608002 India.. 